\title{
Tesis de la carga teórica de la observación y constructivismo
}

\author{
THEORY-LADEN THESIS AND CONSTRUCTIVISM
}

Mg. Juan Carlos Aguirre-García (jcaguirre@unicauca.edu.co) Departamento de Filosofía, Facultad de Ciencias Humanas y Sociales, Universidad del Cauca (Popayán, Colombia)

Dr. Luis Guillermo Jaramillo-Echeverri (ljaramillo@unicauca.edu.co) Departamento de Educación Física, Facultad de Ciencias Naturales, Exactas y de la Educación, Universidad del Cauca (Popayán, Colombia)

\begin{abstract}
The Thesis of Theory-Laden [TTL] holds that is not possible a neutral observation. From this thesis, some philosophers have inferred that the facts, i.e., the subject's independent reality, do not exist or that they are social constructions only. The aim of this paper is assess if TTL necessarily implies a constructivist point of view or if, conversely, we can still speak about the reality. In order to do this, we will clarify these terms: "the theoryladenness of observation" and "constructivism". Then, we will argue that although TTL is possible, we are not committed to constructivism, and on the contrary, TTL can be an interesting frame to the realistic thesis. Finally, we will point out the impact this had has when evaluating many epistemological proposals.
\end{abstract}

Keywords: theory-laden, constructivism, observation, reality, Gergen.

\section{Resumen}

La tesis de la carga teórica de la observación [CTO] sostiene que no es posible una observación neutral. A partir de esta tesis, algunos filósofos han derivado que los hechos, o mejor, la realidad independiente del sujeto, no existen o que son solo construcciones sociales. El objetivo de este artículo es evaluar si CTO implica necesariamente una visión constructivista o si, por el contrario, permite seguir hablando de realidad. Para ello, se aclararán los conceptos de "carga teórica de la observación" y de "constructivismo". Posteriormente, se defenderá que pese a la plausibilidad de CTO, no estamos comprometidos a adoptar el constructivismo, antes bien, CTO puede ser un interesante marco para las tesis realistas. Finalmente, se señalarán algunas repercusiones que esto tiene a la hora de evaluar propuestas epistemológicas.

Palabras clave: carga teórica, construccionismo, observación, realidad, Gergen.

\section{Introducción}

Se intentará dar respuesta negativa a la pregunta que le da el título a este artículo (1); es decir, se tratará de mostrar que, aún si fuera cierta la tesis de la carga teórica de la observación, no estamos obligados a asumir el constructivismo (2). Antes de pasar a exponer cada uno de los términos de estas afirmaciones, se señalará, brevemente, por qué creemos que este es un asunto relevante para las cuestiones epistemológicas contemporáneas. 
En primer lugar, son múltiples los casos en los que escuchamos la expresión "construcción social". No vamos a presentar ejemplos. Valga recordar que Hacking (1999) ha hecho una lista indicativa de títulos de trabajos académicos en la que cubre, sin agotar, todo el abecedario; por consiguiente, parece necesario aclarar filosóficamente el sentido de tal expresión. En segundo lugar, dicha expresión parece haber gozado de gran estima a lo largo de la historia de la filosofía, entremezclándose con grandes desafíos a la racionalidad, por ejemplo: el relativismo, el escepticismo, el subjetivismo; por tanto, la consideración del constructivismo obliga a recoger discusiones de vieja data, presentes en muchos debates filosóficos, considerándolas a la luz de las discusiones presentes. Finalmente, la reflexión sobre el constructivismo es relevante porque ha sido el suelo nutricio de varias discusiones en ciencias humanas y sociales; por consiguiente, en el contexto de la interdisciplinariedad, la filosofía puede aportar en la clarificación de los fundamentos epistemológicos que tales ciencias adoptan sin darse cuenta, en ocasiones, de las implicaciones que esto conlleva.

Aunque el tema tiene muchas aristas y una exposición como la presente puede correr el riesgo de reduccionista, nos atrevemos a sugerir algunos puntos nodales que, apenas insinuados, permitirán respaldar, así sea esquemáticamente, nuestra posición. En primer lugar, se sintetizará lo que, según la literatura, se define como tesis de la carga teórica de la observación. Posteriormente se recurrirá a uno de los exponentes fundamentales del constructivismo social, Kenneth Gergen (Yang y Gergen 2012), para encontrar, en sus propias palabras, la definición de construccionismo. En segundo lugar, se señalarán dos objeciones a la doctrina de la carga teórica de la observación: el mecanismo de percepción "modular" de Fodor (1984) y la teoría del ver no-conceptual de los objetos expuesta por Dretske, pero para la presente exposición nos remitimos a la reconstrucción que de ella hace Nola (2003). Por último, respaldados en las tesis precedentes, mostraremos que no hay motivos empíricos concluyentes para hablar de carga teórica de la observación; además, si los hubiera, no toda observación caería en esta pretensión y, por tanto, podría apelarse coherentemente a un realismo directo o crítico.

\section{Carga teórica de la observación y constructivismo: definiciones mínimas}

Se atribuye a Don Ramón de Campoamor el popular verso: "En este mundo traidor, nada es verdad, ni mentira, todo es según el cristal con que se mira". Podría decirse que esta humorada (3) recoge lo fundamental del planteamiento de la carga teórica de la observación. En síntesis, quien determina la verdad de algo no es la observación, entendida como experiencia sensorial, sino la teoría en la que un hecho es interpretado.

Si bien, como se ha afirmado, esta es una discusión con largo linaje en filosofía, el contexto en el que la discusión sobre la observación ha alcanzado un reconocido relieve ha sido el debate sobre la ciencia. El éxito de la física moderna y la postulación de la observación como garante de tal éxito -por encima de las deducciones medievalesfue haciendo de la empresa científica una construcción sólidamente levantada sobre fundamentos seguros. Cuando en el siglo XX la ciencia se convierte en un objeto de teorizar filosófico, algunos filósofos de entonces, pertenecientes a la hoy llamada "concepción heredada", radicalizaron la función de la observación en ciencia. Parafraseando a Ayer, el filósofo André Kukla sostiene: "de acuerdo con los primeros (y más radicales empiristas) proponentes de esa escuela filosófica, un enunciado carece de significado a menos que pueda ser traducido, o reducido, a lenguaje de observación -un lenguaje que consiste de términos que describen solo propiedades observables de cosas observables. [Y agrega, a guisa de ejemplo] "Los enunciados acerca de los electrones inobservables podían ser reducidos a enunciados acerca de los rastros sobre placas fotográficas; los enunciados acerca de los estados mentales inobservables eran traducidos a enunciados acerca de la conducta observable" (Kukla 2008:396). De acuerdo con esto, los enunciados de las teorías científicas tenían que ser lógicamente 
equivalentes a enunciados de observación; de igual modo, la elección de una teoría tenía que estar en consonancia con la adecuada consecuencia observacional.

Esta concepción, englobada por las mayorías como "positivista" (a propósito, algunos aún creen, erróneamente, que esta es la visión que adoptan todos los filósofos de la ciencia), empezó a desmoronarse, principalmente, a partir de investigaciones surtidas en el terreno de la psicología, en especial, de los hallazgos empíricos emanados de la psicología de la forma. En la década del sesenta del siglo pasado, los filósofos Hanson (1977) y Kuhn (1996), entre otros, recogieron lo producido por la investigación psicológica y comenzaron a revaluar el papel de la observación en el proceso de investigación científica. Hanson, del cual Kuhn tomó varias de sus ideas, presenta multitud de casos de la historia de la ciencia, en los cuales dos personas, por ejemplo Tycho y Kepler, a pesar de ver el mismo fenómeno, tienen una experiencia distinta de ella, la interpretan de modo diferente. De ahí concluye Hanson que: "decir que Kepler y Tycho ven la misma cosa al amanecer solo porque sus ojos son afectados de un modo similar es un error fundamental. Existe una gran diferencia entre un estado físico y una experiencia visual" (Hanson 1977:84). De un modo más general plantea: “los receptores de señales ópticas, no importa lo sensibles y exactos que sean, no pueden proporcionar todo lo que se necesita para observar [por ejemplo] la resistencia eléctrica. Se presupone también un conocimiento; la observación científica es, por tanto, una actividad "cargada de teoría»" (Hanson 1977:13). Kuhn dio un paso más allá de Hanson: no se trata de meras diferencias en la interpretación de los datos; las observaciones responden a un paradigma y cuando cambia el paradigma, "los datos mismos han cambiado [...] Después de una revolución, los científicos trabajan en un mundo diferente” (Kuhn 1996:135).

En consecuencia, la tesis de la carga teórica de la observación es una reacción, sustentada en la psicología empírica, contra las pretensiones de observación neutral postuladas por algunos filósofos, especialmente los de la concepción heredada. Tal tesis sostiene que existe una diferencia entre las "experiencias de datos sensoriales" y las "interpretaciones ex post facto de lo que se ve"; ahora bien, tales interpretaciones responden al trasfondo teórico que tenga el observador. Por tanto, no habría posibilidad de encontrar "hechos desnudos"; todos estarían arropados por, o dependerían, del "cristal con que se mira".

Muchos académicos, respaldados en la tesis de la carga teórica de la observación, extrajeron diversas conclusiones. Algunas de ellas plantean que, al no haber aproximación neutral a los hechos, no hay posibilidad de toparnos con la 'Realidad' (con ' $R$ ' mayúscula), sino que esta se configura a partir de los conceptos o marcos de los individuos o los sujetos; por tanto, hay tantas realidades cuantos sujetos o comunidades haya. Por consiguiente, tampoco podremos hablar de 'Verdad' (con ' $V$ ' mayúscula), sino de verdades dependientes de.... (los puntos suspensivos pueden llenarse con: el individuo, una comunidad $X$, los que detentan el poder, las mujeres, los movimientos $Y$, etc.).

En la contemporaneidad, uno de los exponentes más radicales de este planteamiento es el movimiento denominado "construccionismo social". El argumento central fue recogido por Hacking de la siguiente manera (valga decir que solo recogeremos lo que él enuncia como formulación 1 . Las formulaciones 2,3 y 0 se dejan fuera de consideración): "No es necesario que $X$ haya existido, ni es necesario que sea como es. $X$, o $X$ como es en el momento presente, no está determinado por la naturaleza de las cosas; no es inevitable" (Hacking 1999:6). Teniendo clara esta formulación, vamos a considerar a uno de los difusores más prolíficos del construccionismo social, el psicólogo social Kenneth Gergen (4). 
Según Gergen, el construccionismo social es una meta-teoría del conocimiento, tal y como lo puede ser el empirismo; sin embargo, esta meta-teoría es, a nuestro modo de ver, muy sui generis pues sostiene que "no hay una verdadera explicación de verdad, no hay verdad con ' $V$ ' mayúscula, tampoco hay una objetividad que se oponga a una subjetividad. Es decir, que la meta-teoría funciona como un tipo de sombrilla bajo la cual todas las explicaciones del mundo [...] se pueden colocar" (Yang y Gergen 2012:127). Obviamente, si el construccionismo es una teoría del conocimiento, digamos, sin lo que tradicionalmente se ha entendido por conocimiento, entonces nos preguntamos ¿qué tipo de preguntas trata de responder esta teoría? En efecto, Gergen aclara que este nuevo "paradigma" aborda otro tipo de cuestiones, por ejemplo: “¿cómo y para quién es útil lo que estás haciendo?”, o “¿cuáles son las implicaciones socio-políticas de tomar seriamente la realidad propuesta?” Las características expuestas por Gergen dan a entender que este nuevo paradigma es más flexible y comprehensivo que los anteriores, toda vez que puede convivir con ellos en tanto no es un sistema de creencias y, en consecuencia, no entraría en conflicto con un sistema de creencias opuesto. Al respecto dice Gergen: "las ideas construccionistas deben mirarse de acuerdo con las prácticas a las que ellas invitan; ensayarlas y ver lo que ocurre. En este sentido, son más como la música o la poesía, que como la biblia o la ontología fundamental” (Yang y Gergen 2012:129).

Si el construccionismo social es una meta-teoría del conocimiento sin conocimiento, al estilo clásico, entonces, ¿qué es? Pareciera que el construccionismo es lo que hoy se conoce como una visión de mundo (worldview), una especie de atmósfera que envuelve cada rincón de la existencia. Leamos a Gergen que es, incluso, bastante poético: "Desde mi perspectiva, el construccionismo no es solo un accidente histórico. Es una forma más refinada de una sensibilidad global emergente; es decir, cada vez el mundo se está dando cuenta de las múltiples perspectivas, especialmente debido a las tecnologías de la comunicación. Como nos hemos dado cuenta de las multiplicidades en las perspectivas y los valores, así como del problema de determinar 'qué es verdadero o correcto', los fundamentos para el construccionismo ya están establecidos. Así pues, de algún modo, el construccionismo es simplemente una articulación -o manera de clarificar- algo que está ya en el mundo como un movimiento. ¿Ves lo que quiero decir? Es como la conciencia que necesita las palabras del construccionismo para llegar a ser consciente de sí misma y su potencial” (Yang y Gergen 2012:131).

Para completar la presentación del construccionismo, valga resaltar dos elementos más. El primero es que, para el construccionista, todo conocimiento está cargado de valor; por tanto, no deberíamos preguntarnos por lo que dijo alguien, sino por el valor práctico que tiene adoptar su planteamiento, para qué y para quién es útil, y si podría ser de otro modo, es decir, si podría explorarse otras posibilidades. El segundo elemento, que se desprende de la última pregunta, tiene que ver con la importancia de las posibilidades para el construccionista, la cual es inversamente proporcional al sentido de la realidad para el realista. Dice Gergen: "la tendencia hacia el realismo [...] restringe nuestras posibilidades. Estamos tratando aquí con convenciones al hablar y ellas podrían ser de otro modo" (Yang y Gergen 2012:132).

En síntesis, el construccionismo social es una teoría del conocimiento cuyo planteamiento central es que no hay una realidad, entendida como corte de apelación definitiva, sino que hay múltiples posibilidades, dependiendo de los modos como un individuo o colectividad interpreten los distintos fenómenos a los que se enfrentan.

\section{Dos contraargumentos a la tesis de la carga teórica}

La tesis de la carga teórica de la observación ha gozado, como hemos visto, de amplio respaldo psicológico y filosófico; igualmente, ha sido acogida entusiastamente por ciertas disciplinas humanas: sociología, antropología, psicología. En ocasiones, esta tesis se ve como incuestionable, más aún, como un presupuesto obvio a la hora de discutir sobre el conocimiento. En el presente apartado se consideran dos críticas a la tesis de la carga teórica de la 
observación. Posteriormente, en el apartado tres, expondremos algunas ideas que, tomadas de la crítica a la tesis de la carga teórica de la observación, nos permitirán concluir que no estamos condenados a adoptar el construccionismo y, de paso, que podemos adoptar un realismo epistemológico (5).

En primer lugar consideraremos las tesis de Jerry Fodor, consignadas en su artículo La Observación Reconsiderada, donde discute la manida distinción entre observación e inferencia (6). Este autor es un importante referente pues reta la tesis en el mismo terreno en el que se enunció, es decir, en la psicología, aunque no se restringe a ella. El propio Fodor reconoce que los argumentos a favor de la distinción observación / inferencia son a menudo tomados tanto del lenguaje ordinario como del holismo del significado; sin embargo, pese a que los enuncia, se centra en lo que él denomina "argumentos psicológicos de facto" (Fodor 1984:25). Solo estos serán tenidos en cuenta en esta presentación. La reconstrucción del argumento contra el que Fodor se pronuncia es presentada por él mismo de la siguiente manera: "La percepción, de acuerdo con esta explicación, es el proceso en el que un organismo asigna causas distantes probables a los estímulos próximos que se encuentra. Lo que da la solución a los problemas perceptuales, distintos a la mera rutina, es el hecho que, como una cuestión de principio, cualquier modelo dado de estímulo próximo es compatible con una gran variedad de causas distantes; hay, podríamos decir, muchos mundos posibles que proyectarían un modelo de excitación dado en el mecanismo sensorial de un organismo. Para ver el proceso mental que está en el medio de la percepción como inferencias, es necesario ver las inferencias como no-demostrativas [...] Es valioso enfatizar la moral de esto: lo que media la percepción es una inferencia de efectos a causas" (Fodor 1984:30).

La pregunta obligada que se hace Fodor es la siguiente: "Si, en general, hay muchas soluciones distantes compatibles con el problema perceptual que plantea un modelo sensorial dado, ¿cómo es posible que la percepción podría ser tomada alguna vez como unívoca (por no decir verídica)?" (Fodor 1984:31). Pareciera que la única posibilidad es sucumbir a la tesis según la cual la información sensorial es interpretada con referencia a las teorías que tiene como trasfondo quien percibe. Pero en este punto Fodor encuentra un problema: "si debemos impresionarnos por el grado en que la percepción es interpretativa, contextualmente sensible, lábil, respondiendo al conocimiento de trasfondo y todo eso, también deberíamos estar impresionados por el grado en el cual a menudo son testarudas y recalcitrantes" (Fodor 1984:33). Fodor está haciendo referencia a la clásica ilusión MullerLyre (Figura 1), la cual es comúnmente usada para sustentar la tesis de cómo la información de trasfondo influye en la percepción.

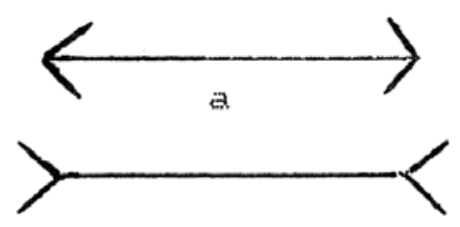

b

Figura 1: La ilusión Muller-Lyre

Fodor reinterpreta esta figura señalando que una persona con conocimientos básicos, sabe que ambas líneas tienen la misma longitud, pese a que la línea b parezca más larga. Así pues, si a alguien ilustrado se le pregunta cuál de las dos líneas ( $a$ o b) es más larga, responderá inmediatamente: “ambas miden lo mismo"; sin embargo, en la percepción sigue apareciendo la figura b como más larga. Ante esto se pregunta Fodor: ¿Por qué ESA parte de la teoría de trasfondo no influye en la percepción?, es decir, ¿por qué sin saber que las líneas son de la misma 
extensión terminamos pensando que las líneas tienen la misma longitud? Antes que un ejemplo de cómo las teorías influyen en nuestra percepción, Fodor está demostrando que la forma como vemos el mundo está particularmente no afectada por lo que uno conoce de él. La conclusión de Fodor es un punto intermedio entre la neutralidad pretendida por los positivistas y la imposibilidad de acceder a los hechos postulada por los constructivistas. Concluye Fodor: "es perfectamente posible [...] apuntar hacia un punto medio: [...] estar de acuerdo [...] en que hay un sentido importante en el que la observación es un tipo de inferencia, pero también de acuerdo [...] en que hay, en la percepción, un aislamiento radical en cómo se ven las cosas de los efectos de todo lo que uno cree" (Fodor 1984:35). Esta segunda alternativa, la del aislamiento, es abordada por Fodor bajo la tesis del mecanismo perceptual modular, lo cual sería asunto de otra trabajo; baste decir que la evidencia empírica que respalda la tesis de la carga teórica, es la misma que respalda su opuesto; en tal sentido, habría buenas razones para creer que no toda observación está cargada de teoría.

El segundo argumento contra la tesis de la carga teórica es tomado de Dretske (7), y se dirige hacia una de las aristas que se desprenden de tal tesis, el conocido argumento de la inaccesibilidad de la realidad, según el cual: "nunca podemos comparar ni nuestras experiencias de, ni nuestras creencias acerca de la realidad con el modo como la realidad es, pues en orden a mirar si nuestras experiencias o creencias corresponden con la realidad, siempre otras experiencias o creencias deben intervenir" (Nola 2003:131). De este modo, nunca podremos tener conocimiento directo, más aun, nunca podremos tener ningún conocimiento de cómo es la realidad. El presente contra-argumento retará la posición de que "no podemos comprobar nuestra experiencia con la realidad" (Nola 2003:136).

La teoría de la percepción de Dretske comienza con la consideración de la experiencia de ver. Lo primero que hace Dretske es establecer una diferencia entre un 'ver epistémico', que involucra un juicio o una creencia, y un sub o pre-ver, al cual llama 'ver no-epistémico'. Por ver no-epistémico Dretske quiere decir: "una condición negativa en la que una persona puede ver algún objeto X pero sin necesidad de tener alguna creencia en orden a ver X" (Nola 2003:154). La exposición de esta distinción es hecha por Nola de la siguiente manera: "Su tesis general [de Drestke] acerca del ver no-epistémico tiene que ver con casos en los que el verbo 'ver' no toma una cláusula-que (thatclause) como su objeto gramatical, como en 'La persona P ve que es un gato', o 'P ve que el niño fue secuestrado', o 'P ve que el fósil es del miembro de un antílope', etc. Más bien, tiene que ver con casos en los que el verbo 'ver' toma como objeto gramatical un nombre o una descripción como en: 'P ve el gato', o 'P ve el secuestro del niño', o 'P ve el miembro del fósil de un antílope', etc., respectivamente. Su tesis acerca del ver no-epistémico gira sobre la afirmación correcta de que casos de ver no-epistémico, como los últimos, no involucran casos de ver epistémico, como los primeros. De hecho, su planteamiento es mucho más fuerte que este en la medida en que descarta cualquier creencia como requisito necesario de ver no-epistémico" (Nola 2003:154).

Un caso traído recurrentemente por los defensores de la tesis de la carga teórica es el caso del nativo al que se le coloca, digamos, frente a algún edificio occidental (por ejemplo, la Catedral de Notre Dame). Según ellos, el nativo no ve la Catedral de Notre Dame, toda vez que 'Catedral' es un nombre dado a edificios destinados al culto, y 'Catedral de Notre Dame' es uno de esos edificios que se empezó a construir en 1163. Ninguno de estos conceptos, aducen, dice algo al nativo (8).

La distinción establecida por Dretske nos permite afirmar que el nativo ve la catedral sin ver que o creer que es una catedral. En palabras de Dretske: "la ignorancia de X no perjudica la visión de X" (Nola 2003:155). Así pues, el nativo puede creer que, por ejemplo, la catedral es un espíritu extraño, un Dios occidental, un espejismo o una alucinación; sin embargo, ninguna de estas creencias que pudiera tener el nativo afectan de algún modo el hecho 
de que el nativo ve 'no-epistémicamente'. Sin embargo, para el ver no-epistémico, Dretske establece unas condiciones, a saber, (1) que la catedral esté en el campo visual de P y (2) que sea un ítem distinto de otros ítems en el campo visual.

En conclusión, podemos ver que hay un tipo de ver objetos (e incluso hechos tales como el gato estando en el tapete) que no involucran ninguna creencia acerca del objeto (o el hecho). Y aunque las creencias también involucren conceptos, podemos decir que hay un ver no-conceptual. Podemos también hablar apropiadamente de un contenido visual, o un contenido perceptual, pero no quiere decirse por 'contenido' algo proposicional; es contenido no-conceptual. Es más bien un contenido visual, dado en gran parte por las distintas propiedades visuales de (objeto al final del cono del campo visual de P) y sus alrededores (Nola 2003:157).

\section{¿Cuál es la moraleja de esto para el debate sobre el constructivismo?}

Los argumentos presentados aquí contra la tesis de la carga teórica de la observación (aunque necesitados de más desarrollo y crítica), darían pie para poner en cuestión una de las tesis favoritas del construccionismo, en especial del epistemológico, a saber, que en vista de que no hay hechos a los cuales apelar (en tanto todos están cargados de teoría), no habría tampoco un criterio último para determinar qué cuenta como conocimiento; es decir, estaríamos condenados a decir, con igual valor de verdad, que cuando Kenneth Gergen da un pequeño salto, Liping Yang interpreta que Gergen vuela, mientras que nosotros, en nuestra corta imaginación, solo vemos a Gergen dando un pequeño salto (9).

Pese a que esto es meramente en el plano de lo anecdótico, las conclusiones a las que llega el constructivismo son mucho más audaces (10): el que la observación sea dependiente de la teoría, implicaría que tenemos que admitir que el conocimiento es desesperanzadoramente subjetivo. Si, en el mejor de los casos, sostuviéramos que hay una mezcla indiscernible entre observación y teoría, tendremos que concluir que cualquier observación o reporte de observación no puede operar objetivamente, ya que no puede fungir como árbitro neutral capaz de hacerse a un lado y dar un juicio libre de prejuicio. Si la observación no puede ser objetiva, la elección de lo que cuenta como conocimiento o como teoría científica, no podrá hacerse sino a partir de gustos u opiniones personales. En definitiva, tal y como muchos discursos se ufanan de replicar, la objetividad del conocimiento en asuntos tanto de observación como de teoría, es un mito que ha entrado en bancarrota.

La moraleja que tratamos de extraer de este asunto intenta zafarlo de las polaridades en las que se halla. Pese a que los contra-argumentos son razonables, es decir, socavan la solidez de la tesis de la carga teórica, quizás pueda parecer que en este terreno la discusión no está agotada (11). Algunos podrían insistir en la tesis de la carga teórica de la observación. Quizás nosotros mismos la respaldemos, es decir, coincidamos en que la mayoría de las observaciones interesantes en el proceso del conocimiento superan el carácter modular, según Fodor, o noepistémico, según Dretske; sin embargo, de ahí no se siguen las conclusiones que esgrimen los construccionistas. Queremos centrarnos sobre todo en dos elementos que aún podrían salvaguardarse:

1. Que la observación esté cargada de teoría no implica que sea imposible la objetividad del conocimiento, es decir, no implica que todo conocimiento sea construido a la medida del sujeto o de la comunidad en el que se enuncia. $A$ lo sumo podemos concluir que la observación es falible, o sea, que la observación no puede darnos un fundamento cierto y seguro. En palabras de Sankey, “los presupuestos teóricos que guían la observación pueden hacer que evidencia importante sea pasada por alto, así como pueden hacer que a la evidencia irrelevante se le dé un significado inmerecido, [además] la contaminación de la actividad perceptual por la teoría significa que un hecho observado podría ser malinterpretado debido a la influencia de la teoría sobre la interpretación del hecho. Y la 
influencia de la teoría sobre el lenguaje observacional significa que el significado de reportar un hecho observado contiene la posibilidad del mal reporte de los hechos" (Sankey 1999:206).

2. Así pues, la objetividad no requiere que la observación sea inmune a la teoría: "lo que se requiere para que la recolección de los hechos empíricos sea objetiva es más bien que haya procedimientos que permitan a distintos observadores llegar a un acuerdo sobre los hechos" (Sankey 1999:206). Tales procedimientos, sean técnicas experimentales o instrumentos, permiten resultados repetibles, no dependientes del estado subjetivo. Así pues, Tycho, el ejemplo favorito de Hanson y muchos de los construccionistas contemporáneos, si bien interpretaba el sol de modo distinto a como lo hacía Kepler, pudo haber sido persuadido, mediante el uso de instrumentos a los que era tan aficionado, de que, en efecto, la tierra se mueve, hecho que ningún construccionista serio se atrevería hoy a negar.

\section{Notas}

1. Este artículo es un avance del proyecto de investigación: "Las retóricas del construccionismo epistemológico. Las concepciones de Ciencia, Realidad y Racionalidad de los Investigadores Sociales de la Universidad del Cauca" (ID 3376), financiado por la Vicerrectoría de Investigaciones de la Universidad del Cauca (Popayán, Colombia). Los autores dan expreso crédito y mención a la Universidad del Cauca por los espacios y tiempos permitidos para el desarrollo de estas reflexiones.

2. Al igual que lo señalamos en Aguirre y Jaramillo (2010), pareciera que, de acuerdo con los propios promulgadores, el término más preciso sería construccionismo. Sin embargo, la mayoría de los impugnadores de esta propuesta adoptan el término constructivismo (constructivism); en tal sentido, intentaremos utilizarlo en consonancia con la formulación original de cada autor.

3. Por humorada se entiende "breve composición poética que encierra una advertencia moral o un pensamiento filosófico de forma cómica".

4. Es preciso advertir que únicamente nos remitimos a la entrevista concedida por Gergen a Yang, publicada en 2012. Una presentación amplia de las tesis de Gergen, seguida de una apreciación crítica del construccionismo social puede encontrarse en López (2013).

5. Podría decirse que el realismo epistemológico ha sido una de las corrientes que más ha confrontado al construccionismo y advertido acerca de sus consecuencias. Una aproximación, desde el contexto de la filosofía de la ciencia, se encuentra en Aguirre y Jaramillo (2010). Sin embargo, hemos visto recientemente cómo el debate se ha ido ampliando a esferas en las que tradicionalmente no se había considerado directamente. Como ejemplo de la reciente respuesta realista en el terreno de las ciencias humanas y sociales, remitimos a Ferraris (2012a y 2012b), así como la airada respuesta de Vattimo (2012). Igualmente, pueden verse los trabajos de López (2013) y Gaete (2013). Estos trabajos recientes nos hacen sospechar que el debate entre realismo y construccionismo, ahora expresamente en el terreno de las ciencias sociales, se ha renovado y son muchos los argumentos que todavía faltan por considerar y revaluar al respecto.

6. Para Fodor existe una equivalencia entre los términos "inferencia" y "resolver problemas".

7. Como se advirtió desde el inicio, todas las referencias serán tomadas de Nola (2003), el cual se apoya fundamentalmente en Dretske (1969).

8. El caso del nativo lo parafraseamos del ejemplo de "Omai, el tahitiano que llevó James Cook a Europa en su segundo viaje al Pacífico, en 1770", relatado por Nola (2003:154).

9. Nos remitimos aquí a una extraña aseveración hecha por Gergen en la entrevista, la cual reproducimos literalmente: "Si los críticos dicen: 'tú quieres decir que los seres humanos pueden volar: eso es ridículo'. Puedo responder: 'bien, imira!' Luego doy un pequeño salto. Los críticos dicen: 'Eso no es volar', y respondo: 'Bien, a eso es a lo que yo llamo volar'. Si llamo a eso 'volar', puedo volar" (2012:132).

(10) En este punto conclusivo seguimos muy de cerca los análisis realizados por Sankey (1999).

(11) Aunque creemos que estos argumentos son promisorios. Los reconocidos construccionistas científicos (pertenecientes al Programa Fuerte en Sociología de la Ciencia, Barry Barnes, David Bloor y John Henry, en su texto Scientific Knowledge: A Sociological Analysis (1996), refiriéndose a la observación científica), parecen aceptar sin 
discusión la contundencia de los argumentos de Fodor. Por ejemplo, a la tesis de Hanson la denominan Falacia Kundgabe. Además, después de examinar las tesis de Fodor y Churchland concluyen "nuestra opinión es que, haciendo un balance, la posición defendida por Fodor está mejor respaldada por la evidencia” (Barnes, Bloor y Henry 1996:12).

\section{Bibliografía}

Aguirre, J. y Jaramillo, L. 2010. La ciencia entre el objetivismo y el construccionismo. Cinta moebio 38: 72-90.

Barnes, B; Bloor, D. y Henry, J. 1996. Scientific knowledge: a sociological analysis. London: Athlone.

Dretske, F. 1969. Seeing and knowing. London: Routledge and Kegan Paul.

Ferraris, M. 2012a. Manifesto del nuovo realismo. Bari: Laterza \& Figli Spa.

Ferraris, M. 2012b. A new realist approach to hermeneutics. Phainomena 21(82-83): 67-84.

Fodor, J. 1984. Observation reconsidered. Philosophy of Science 51(1): 23-43.

Gaete, A. 2013. ¿Es sostenible el constructivismo radical? Cinta moebio 46: 1-8.

Hacking, I. 1999. The social construction of what? Cambridge: Harvard University Press.

Hanson, N. R. 1977. Patrones de descubrimiento: observación y explicación. Madrid: Alianza Editorial.

Kuhn, T. S. 1996. The structure of scientific revolutions. Chicago: Chicago University Press.

Kukla, A. 2008. Observation. En: S. Psillos y M. Curd (eds.). The Routledge companion to philosophy of science. London: Routledge, pp. 396-404.

López, P. 2013. Realidades, construcciones y dilemas. Una revisión filosófica al construccionismo social. Cinta moebio 46: 9-25.

Nola, R. 2003. 'Naked before reality; Skinless before the absolute': A critique of the inaccessibility reality argument in constructivism. Science \& Education 12: 131-166.

Sankey, H. 1999. The theory-dependence of observation. Cogito 13(3): 201-206.

Vattimo, G. Realism in two pieces. Phainomena 21(82-83): 85-90.

Yang, L. y Gergen, K. 2012. Social construction and its development: Liping Yang interviews Kenneth Gergen. Psychological Studies 57(2): 126-133.

Recibido el 20 Feb 2013

Aceptado el 4 May 2013 